\title{
La educación inicial argentina y española en el contexto de cuarentena: análisis de casos
}

\author{
Argentine and Spanish initial education \\ in the context of quarantine: case analysis
}

María Ferrero Guindel ${ }^{1}$

Sheila Frutos Quintero ${ }^{2}$

Sara García Castellanos ${ }^{3}$

Sara García Núñez ${ }^{4}$

Universidad Camilo José Cela

Julia Pereira de Lucena ${ }^{5}$

Universidad de Ciencias Empresariales y Sociales

Recibido: 30.11 .2020

Aceptado: 14.12.2020

\section{Resumen}

Hasta el año 2020, los cambios a la hora de educar en la práctica solían producirse de forma paulatina y concreta en los distintos niveles educativos. En el nivel inicial, la inclusión de tecnologías en muchas oportunidades generó resistencias. En la actualidad, sabemos que es necesario repensar las bases teóricas y las prácticas que hacen referencia a la educación ya que se han produciendo cambios importantes y vertiginosos en el contexto de cuarentena obligatoria generado por el COVID- 19. Realizaremos un análisis de casos de propuestas

\footnotetext{
${ }^{1}$ mafegui92@hotmail.com

2 espana.sheilacachito@ hotmail.com

${ }^{3}$ sara.garcia.castellanos@gmail.com

${ }^{4}$ mafegui92@ hotmail.com

${ }^{5}$ mjulialucena@gmail.com
} 
educativas generadas y desarrolladas por tres instituciones de nivel inicial de Argentina (Ciudad Autónoma de Buenos Aires y ciudad de Ezeiza) y España (Madrid) en donde se reflejan dichos cambios abruptos. Relacionaremos estos casos con autores clave en campos tales como la Didáctica del Nivel Inicial y la Tecnología Educativa.

Palabras clave: Nivel inicial, Tecnología educativa, Cuarentena, Educación virtual.

\begin{abstract}
Until 2020, educational changes in schools were made gradually and specific to the corresponding educational level. In Early Childhood Education, the incorporation of technologies has been generating some resistance. Nowadays, due to COVID 19, the situation of the global pandemic and the quarantine have produced mandatory changes in the theoretical bases and educational practices. We will analyze different educational proposals made in three Early Childhood Education schools, two of them in Argentina (Autonomous city of Buenos Aires and Ezeiza City) and the other in Spain (Madrid). We will connect these cases with authors in the field of Early Childhood Education and Educational Technology.
\end{abstract}

Keywords: Early Childhood Education, Distance learning, Technology, Quarantine and Elearning.

\title{
Introducción
}

En el presente artículo, nos proponemos reflexionar acerca de la inclusión de las tecnologías de la información y la comunicación (TIC) en instituciones educativas de dos ciudades argentinas (Ciudad Autónoma de Buenos Aires y Ezeiza) y una española (Madrid) en el contexto de la cuarentena por el COVID - 19 que ha iniciado en marzo de 2020.

Analizaremos cómo ha sido el uso de las TIC y las TAC durante la cuarentena, aclarando que, si bien se toman y retoman algunos elementos de la opción educativa conocida como 
educación a distancia, en este caso no hubo elección y su desarrollo fue obligatorio. Además, se trata de un modelo distinto e inédito dado que no se encontraron antecedentes en el mundo contemporáneo y a escala global de este tipo de estrategias de enseñanza y de aprendizaje sostenidas a lo largo de los meses por razones de prevención masiva de la salud de la población. Algunos docentes (Pettinari, 2020) hablan de “educación en emergencia sanitaria”.

Asimismo, analizaremos cómo se han puesto en juego diferentes dimensiones pedagógicas durante la cuarentena. Finalizamos el trabajo con algunas conclusiones e interrogantes, partiendo de la información que tenemos disponible a través de los casos prácticos analizados y los documentos bibliográficos en los que nos hemos basado. Todo ello nos va a permitir arrojar un poco de luz ante esta nueva situación.

\section{1. ¿Nativos digitales? Una mirada crítica acerca de la tecnología}

Desde hace muchos años, diversos autores (por ejemplo, Gay, 1997) afirman que el mundo contemporáneo está condicionado por la importancia de las tecnologías. Esto conlleva a tener en cuenta que las tecnologías forman parte de nuestras vidas, incluyendo en menor o mayor medida a la educación. En ciertos sectores sociales, los niños tienen un uso constante de las nuevas tecnologías: con cada vez mayor frecuencia, vemos a niños de muy corta edad utilizando recursos tecnológicos. Barberá (2010) asegura que la tecnología influye en la educación. Esta es una de las razones clásicas de la fundamentación de la importancia de llevar a cabo el contacto con las tecnologías, desde el nivel inicial, lo que sin duda requiere importantes dispositivos de capacitación docente tanto en su formación inicial como en la continua.

Prensky (2000) distingue entre nativos digitales e inmigrantes digitales y este concepto ha estado en el tapete durante 20 años. Este autor definió a los primeros como aquellos que han nacido en la era digital, considerando las tecnologías como parte de sus vidas y los inmigrantes digitales aquellos que han crecido sin tecnologías y les cuesta adaptarse a ellas. Aunque los alumnos de hoy en día hayan nacido en una época de gran desarrollo y uso de las TIC, no se 
debe dar por hecho que sepan el uso y funcionalidad de las herramientas. Además, nos preguntamos: ¿qué ocurre con el alumnado que no tienen acceso a las tecnologías? ¿se les podría considerar nativos digitales? La falta de accesibilidad puede generar que en algunos casos se produzca una brecha digital, y aunque hayan nacido en una época signada por las tecnologías, no hayan tenido acceso a las mismas debido a la falta de medios o de habilitadas en el uso al interior de su ámbito familiar. Es importante destacar que, en el caso del nivel inicial, los alumnos dependen de manera sumamente directa del acceso y especialmente del uso de las tecnologías que tengan los adultos responsables del hogar.

En los casos en que analizaremos, podemos afirmar que las familias y los alumnos tienen acceso a distintas herramientas tecnológicas, pero no siempre podían utilizarlas adecuadamente. Las instituciones educativas desempeñan un rol importante para que las familias pudieran abordar y acompañar los proyectos que fueron desarrollando las distintas instituciones educativas.

Retomando lo expuesto anteriormente, aunque los niños que llegan hoy en día a las aulas son considerados nativos digitales y muchos de ellos tienen interiorizado el uso de las tecnologías, no debemos dar por hecho que eso sucede en todos los alumnos, pues en algunos casos habrá niños que no tengan acceso a tecnologías o a infraestructuras que les permitan acceder a ellas creando así una brecha digital educativa haciendo referencia tanto al acceso a las TIC como a su uso y apropiación (Chávez, 2017). La cuarentena por pandemia ha generado que se haya tenido que improvisar cómo dar respuesta a la problemática existente porque aquellos alumnos que tenían acceso a las tecnologías se han visto beneficiados mientras que, aquellos que se enfrentaban con limitaciones, se han visto perjudicados, agravándose las diferencias y fomentando una mayor brecha digital. Así, nos preguntamos: ¿se han remarcado o profundizado aún más desigualdades en el nivel de competencia tecnológica del alumnado debido a la educación virtual? ¿se agravan las diferencias de competencia digital entre el alumnado? Tomemos otro caso de análisis: los centros del municipio de Leganés (situado al sur de la ciudad de Madrid) han promovido el reparto de los propios medios del centro (ordenadores portátiles y tablets) a los niños de otros cursos más inferiores que tampoco tenían 
ningún tipo de acceso a las tecnologías desde sus casas evitando así una gran brecha digital que seguramente acrecentaría las diferencias de nivel de cara al curso siguiente ante la imposibilidad de poder acceder a los contenidos trabajados en estos meses.

Como decíamos anteriormente, la brecha digital no solo tiene relación con la disponibilidad de las tecnologías sino también de su uso en la familia. Tomaremos otro caso: un centro situado en la Ciudad Autónoma de Buenos Aires ya contaba con una plataforma educativa online a través de un programa llamado "Aulas en comunión” donde se subían pequeños fragmentos de la vida cotidiana del mismo, pero no tenía un gran uso más allá de compartir fotos con las familias del alumnado. Ante la situación de cuarentena, el equipo directivo del centro decidió emplear esta plataforma para poder seguir enseñando. La plataforma es accesible tanto desde un dispositivo fijo como un ordenador y desde dispositivos móviles, lo que facilitaba el acceso a ella por parte de todas las familias. Asimismo, se subieron tutoriales y vídeos explicativos sobre cómo usar las herramientas que tiene la plataforma para que las familias pudieran tener acceso y hacer uso de ellas con la intención de que ninguna familia quedase sin acceso a las actividades que se subían a la plataforma e intentando así disminuir una posible brecha digital en el uso.

También es destacable que se observa cómo muchas instituciones durante la cuarentena han tenido en cuenta los medios a disposición del alumnado, no solo dentro del aula, si no también fuera con el fin de evitar esta brecha digital. A diferencia del caso comentado en el párrafo anterior, en otro centro (ciudad de Ezeiza, en la Provincia de Buenos Aires) no se contaba con ninguna aplicación o plataforma que permitiera seguir en contacto directo con los más pequeños, ya que la comunicación inicialmente se realizó vía correo electrónico, y se analizó que esto producía falta de contacto sincrónico y personalizado con los niños. En este centro, los docentes tuvieron que hacer una capacitación acerca de la aplicación que iban a usar para mejorar el contacto con los alumnos. Esto pone de manifiesto la necesidad de que los centros cuenten con y/o releven medios tecnológicos a través de los cuales puedan comunicarse tanto con las familias como con los alumnos, adaptándose a las distintas situaciones. 
Por otra parte, en el centro de la Ciudad de Buenos Aires, las capacitaciones ya se habían realizado con anterioridad a la situación de la cuarentena pues, como se mencionó anteriormente, el centro contaba con una plataforma educativa a la que acceder, y los docentes sabían de su uso y herramientas para poder comenzar a trabajar desde el inicio.

\section{EI Nivel Inicial: su historia y parte de su actualidad en el contexto de pandemia}

Según Casablancas (2017), como parte de la historia del Nivel Inicial, se han tenido en cuenta algunos modelos previos funcionales, como, por ejemplo:

John Dewey (1859- 1952, EEUU), referente de la Escuela Nueva, basado en una pedagogía conocida bajo el nombre de la "doctrina del interés", consideraba fundamental partir del interés del alumno a través de la acción directa.

María Montessori (1870- 1952, Italia), que puso su mirada específicamente en la infancia, el juego y los espacios para aprender. Defendía un ambiente de libertad para el alumnado en el que existiera una variedad de materiales que desarrollasen los sentidos.

Ovide Decroly (1871-1933, Bélgica), centrado en una pedagogía defensora del globalismo y la teoría de los intereses. Sus ideas llevaron a la creación de los "centros de interés" (las actividades deben centrarse en torno a los intereses del alumno).

Rosa Sensat (1873- 1961, Cataluña), como referente de renovación pedagógica y otras rutas hacia la innovación. Algunas de las características básicas de su pedagogía son: una educación personalizada, el paidocentrismo (el alumno es el centro del sistema educativo), el desarrollo de aprendizajes transversales, la formación permanente del profesorado y la reflexión conjunta.

Olga Cossettini (1898- 1987, Argentina) y Leticia Cossettini (1904- 2004, Argentina), quienes creían en la influencia de la comunidad y el entorno para llevar a cabo la enseñanza. 
Todas estas corrientes pedagógicas tienen en común su formación académica profesional, la mirada social y, sobre todo, el hecho de partir de la acción y la visión del sujeto que aprende. Es importante aclarar que estos aspectos han ido retomando y adaptándose a la sociedad actual en la que vivimos introduciendo cambios en las metodologías y recursos que se utilizan en el nivel inicial. Las tecnologías clásicas han sido siempre un elemento presente en el nivel inicial. En cambio, Karsenti y Lira (2009) realizaron un estudio en el que se observó, que los docentes en formación utilizan las TIC de manera regular para planificar sus clases, pero no la llevan a cabo en el aula, es decir, utilizan las TIC como búsqueda de información en internet, pero aún no están preparados y formados para llevarla a la práctica en el aula. Lo importante cuando hablamos del uso de las tecnologías en el aula, es plantearse el motivo por el cual las usamos y el cómo las usamos en el aula.

En los casos analizados, podemos observar que estas corrientes también toman vida en las propuestas que se llevaron a cabo en la cuarentena como por ejemplo la influencia de las teorías de María Montessori (Foschi, 2020) ya que puede verse cómo las maestras de los grupos se han adaptado a las necesidades de los niños, anteponiendo sus necesidades emocionales o personales y no solo atendiendo a los contenidos curriculares. En la institución de la Ciudad Autónoma de Buenos Aires, se observa cómo los docentes de este centro educativo comienzan a utilizar en mayor medida la plataforma educativa "Aulas en comunión" y también se ha fomentado el uso del correo electrónico para facilitar la comunicación a nivel individual y la resolución de posibles dudas. En la institución de la provincia de Buenos Aires, los docentes comienzan a hacer uso de una aplicación educativa creada de forma específica desde el inicio de la pandemia (antes de la pandemia se comunicaban con las familias por email). En el caso de la institución pública del barrio sureste de Madrid, se comienza a hacer un mayor uso de la plataforma virtual "EducaMadrid", una plataforma a nivel autonómico donde se incorporan los diferentes proyectos educativos (a través del blog de aula) y el centro se comunica con las familias.

Así, a partir del cierre de las escuelas por el aislamiento preventivo y obligatorio, observamos diferentes formas de organización, haciendo uso de las tecnologías, estos tipos de proyectos 
son buenos recursos para seguir manteniendo el contacto con los alumnos ya que, las clases no se han suspendido, simplemente se ha cambiado la forma de enseñar y la manera de comunicarnos. En el Nivel Inicial es primordial seguir manteniendo el contacto tanto con el alumnado como con sus familias, por ello, a través de las plataformas como "Aulas en comunión" o EducaMadrid se apuesta por seguir manteniendo el contacto de los docentes con el alumnado mediante videollamadas, vídeos, audios, cuentos narrados, etc. Es una experiencia que nos mantiene cerca y gracias a ello se puede conseguir que a pesar de la distancia se siga estando juntos.

Del mismo modo y retomando lo expuesto anteriormente en relación a la historia del Nivel Inicial, puede verse la importancia del juego y de incluir las dinámicas lúdicas en el aula sobre todo durante este periodo de cuarentena, haciendo que los alumnos muestren interés por el aprendizaje de una forma diferente. Actualmente a través de las distintas plataformas de comunicación se están estableciendo dinámicas a través de vídeos y audios para mantener el vínculo, también se están adaptando las planificaciones didácticas para poder llevarlas a cabo de forma virtual sin problemas, se emplean cuentos, etc.

De la misma manera, puede observarse la influencia de la teoría de las hermanas Cossettini a través de las comunidades creadas en los casos que estamos analizando, donde se puede observar la importancia de la participación de todos los miembros de la comunidad educativa en la educación de los más pequeños, desde el equipo directivo y los docentes, hasta las familias.

En el contexto de cuarentena, el cambio drástico en la forma que entendemos el concepto de educación ha tenido por consecuencia el uso de recursos que se encontraban en desuso (como en los casos de Ciudad Autónoma de Buenos Aires y Madrid), y en ocasiones, a generar nuevas plataformas (ciudad de Ezeiza, Argentina) para seguir desarrollando la labor educativa. Como consecuencia de ello, los equipos directivos y los docentes han tenido que aprender, coordinarse y apostar por el uso de las nuevas tecnologías. Por tanto, la capacidad de adaptación y la formación continua jugaron un papel fundamental en el ámbito educativo. Un 
buen maestro debe continuar formándose una vez termine su carrera y a lo largo de toda su vida para dar lo mejor de sí mismo y adaptarse a los cambios (Casablancas, 2014). Debido a que la tecnología avanza a pasos agigantados, sería necesario optar por una formación permanente para poder brindar una educación de calidad acorde a las innovaciones constantes (Tenti Fanfani, 2007, p. 67). Cabe destacar que no solo los docentes siguen formándose y aprendiendo ya que en los tres casos analizados toda la comunidad educativa se ha visto afectada por estos grandes cambios educativos y las familias han tenido que adaptarse a la realidad existente. Teniendo en cuenta a Aguerrondo (2010), destacamos que en la actualidad nos encontramos en una sociedad cambiante en la que muchas propuestas valían ayer pero no nos funcionarán mañana, por lo que será necesario que sepamos adaptarnos a los cambios de la mejor forma posible. Un ejemplo de esto es lo ocurrido en la etapa de cuarentena educativa ya que hubo un cambio rotundo y sin precedentes en una velocidad sorprendente, sin grandes posibilidades de preparación.

\section{Las TIC y las TAC en la cuarentena. Competencias digitales}

Atendiendo a Casablancas (2014), diremos que es importante diferenciar entre dos conceptos clave: por un lado, las TIC, que hacen referencia al componente o aparatos tecnológicos, es decir, las Tecnologías de la Información y la Comunicación. Por otro lado, si nos referimos a las TAC, Tecnologías para el Aprendizaje y los Conocimientos, será para hablar sobre la comprensión y los aprendizajes significativos que se llevarán a cabo en un contexto determinado haciendo uso de las TIC.

A la hora de educar con las TIC y las TAC, podemos hacer referencia a diversos autores como Wedemeyer (citado en citado en Sevilla, Tarasow \& Luna, 2017, p. 182) quien hace hincapié en la individualidad de los alumnos en cuanto a sus tiempos de aprendizaje, respetando los ritmos de cada uno. Y, en segundo lugar, se hace referencia a Moore (citado en citado en Sevilla, et al., 2017, p. 182), el cual considera esencial la autonomía que se les debe proporcionar a los niños a la hora de adquirir sus aprendizajes, delegando poco a poco más responsabilidades en ellos. Además, atenderemos al factor emocional a la hora de introducir

Volumen 1. Número 2. Julio - Diciembre 2021 
las TIC en el aula como un elemento muy importante. En el caso de los docentes, se debe tener en cuenta cómo se sienten a la hora de usarlas, ya que suponen un cambio en el sistema educativo y en la manera de educar con respecto a la forma que se estaba utilizando hasta ahora (miedo, incertidumbre, alegría...) y valorarlo como un aspecto más a tener en cuenta. Por ello es necesario que el propio docente sea capaz de continuar su formación a lo largo de los años, estar en contacto y trabajo con otros docentes, pensar que otra forma de educar es posible, utilizar las emociones como un componente más, preguntar y resolver sus dudas, usar las TIC en el desarrollo de su profesión, entre otras.

Desde una perspectiva más práctica, cuando hablamos de la incorporación de las TIC en el aula, será fundamental el uso que se haga de estas. Existen múltiples tipos de usos que se pueden realizar a partir de una herramienta tecnológica. Según Rolandi (2015), existen cuatro usos fundamentales cuando hablamos de tecnología: consagrados, innovadores, dinámicos y remotos. Es interesante analizar qué tipos de usos de las tecnologías se han dado en los casos analizados, como consecuencia de la pandemia global COVID-19. De esta forma encontramos:

. Usos consagrados: Se refiere a aquellos que, a pesar de los avances tecnológicos, se siguen utilizando de la misma forma, puede variar el soporte, pero no el uso. Este tipo de uso lo podríamos aplicar a cómo las maestras han seguido transmitiendo el contenido y los conocimientos que enseñan a sus alumnos. En el caso de la ciudad de Ezeiza, mientras que antes de la pandemia, se solía hacer a través de reflexiones en la asamblea, murales, etc., en cuarentena el soporte fue digital, con la plataforma ZOOM. Las maestras siguieron haciendo uso de recursos analógicos como los murales, pizarras o mapas conceptuales, por ejemplo, pero el soporte ha cambiado (se usa una pantalla para estar en contacto con el alumno).

. Usos innovadores: son prácticas que ya se realizaban pero que incorporan algunas novedades tecnológicas. Hemos hallado algunas prácticas innovadoras en el caso del barrio sur-este de Madrid, a través de la incorporación de softwares educativos como Edelvives- Moraletra, el 
uso del blog de EducaMadrid, creación de documentos compartidos en la Nube de EducaMadrid por parte de los diferentes equipos educativos, etc.

. Usos dinámicos: se trata de aquellos que podrían hacerse sin tecnología, pero al emplear la tecnología se le da un valor añadido, mejorando las prácticas docentes y realizando mejoras en múltiples formatos. Por ejemplo, en el caso de la institución de la Ciudad Autónoma de Buenos Aires podemos encontrar una biblioteca virtual; se ha creado un repositorio digital de cuentos que suma un valor añadido al aprendizaje, integra la cultura digital y amplía las posibilidades de acceso a cuentos de diferente índole).

. Usos remotos: Se refiere al uso que se hace de los recursos en el pasado, son el recuerdo del uso de tecnologías pasadas usadas en los viejos tiempos. Este caso nos cuesta más verlo reflejado en los casos analizados ya que los centros educativos han apostado por nuevas herramientas tecnológicas y no hemos encontrado prácticas con uso de tecnologías pasadas tales como diapositivas, canciones y cuentos usados con grabadoras, utilización de los medios de comunicación como la televisión o la radio.

Todos estos usos requieren habilidades de lectoescritura por parte de los adultos, las cuales son "uno de los aspectos que deberían ser atendidos tanto en la formación inicial como en la formación continua" (Sevilla, 2016, p. 130). En muchos casos se piensa que con el mayor uso de las TIC ya no serán necesarias estas habilidades "básicas”, pero con el uso de las TIC será aún más necesario utilizar las palabras y la comunicación escrita con el alumnado; en el caso del Nivel Inicial, las familias necesariamente mediatizan dicha comunicación. En el caso de la institución de la ciudad de Buenos Aires, en la sección de 5 años, en el área de Ciencias Naturales se recabó información sobre los animales y luego se realizaron unas fichas para completar los datos obtenidos: dónde viven, qué comen, etc. En el área del arte, cada niño después de realizar su dibujo pudo firmar su obra escribiendo su nombre, del mismo modo que hacen los artistas. En el proyecto sobre la escritora argentina María Elena Walsh, se motivó el proceso de la lectura en estos primeros años de escolaridad. 


\section{Dimensiones pedagógicas y escenas en el Nivel Inicial en cuarentena}

Rosales Estrada (2007, citado en Sevilla, et al., 2017, p. 142) señala las siete dimensiones pedagógicas a tener en cuenta a la hora de analizar una institución educativa:

- Filosófica, atiende al tipo de persona que se quiere formar en el centro. En los casos analizados, puede verse una tendencia a un perfil de colaborar en la formación de ciudadanos y trabajadores autónomos, sociales, con desarrollo de todas sus facetas como individuos;

. Planificación y administración escolar, es decir, qué tipo de estrategias metodológicas se van a trabajar en el centro. En el caso analizado y en la situación particular de cuarentena, estas estrategias estuvieron centradas en metodologías socio- constructivistas unidas a la selección y desarrollo de las TIC como medio de comunicación y de relación tanto como con las familias como con los adultos, dando apoyo no solo educativo si no también personal a las familias del centro;

. Curricular, atiende a los contenidos o experiencias que se van a llevar a cabo. En los centros analizados, se puede observar la gran importancia que se otorga al desarrollo de los aprendizajes significativos y útiles, aplicados a la vida cotidiana, ayudando a los más pequeños en el desarrollo integral de su personalidad;

. Psicológica, teniendo presentes los ritmos a los que debe generarse el proceso de formación. En cualquier centro, esta dimensión debe considerar las particularidades de todos y cada uno de sus alumnos;

. Sociológica, poniendo en entredicho quién debe dirigir el proceso, si el docente o los propios alumnos. En los casos analizados, y dada la excepcionalidad de la situación, los procesos han sido dirigidos por los docentes, a través de los contenidos subidos y explicados en la plataforma, pero siempre teniendo en cuenta los intereses y motivaciones del alumnado; 
. Epistemológica y ética, abordando qué orientación teórica tienen los planes de estudio;

. Evaluativa, analizando los criterios de evaluación que van a ser empleados en los procesos de enseñanza y aprendizaje. En los casos analizados, se observa que hay disposiciones ministeriales en las que se sugiere una evaluación en términos de valoración y seguimiento de los recorridos pedagógicos de los alumnos. Las familias recibirán un informe que dará cuenta de los objetivos y contenidos desarrollados en este periodo.

En los casos analizados, pudimos observar cómo estas siete dimensiones pedagógicas fueron influenciadas directamente por los proyectos institucionales generados a raíz de la cuarentena con fuertes componentes didácticos y tecnológicos la implementación de una serie de estrategias y herramientas didácticas y tecnológicas. En este sentido, deseamos retomar las palabras de Litwin (2008), quien caracterizó distintas escenas en la intersección entre Didáctica y Tecnología: escena de ayuda, escena optimista, escena de producción y escena problemática. A continuación, vamos a describir en qué consiste cada una de ellas y la relación que guardan en los casos analizados:

. Escena de ayuda: esta perspectiva se trata de una concepción radical de la tecnología. La idea es que, a través de su uso, podemos obtener un cambio cualitativo de la enseñanza, ya que la tecnología ayudaría mantener el interés del alumnado, potenciar las capacidades cognitivas, aprendizajes creativos, mejoraría la atención y la anticipación. En los casos analizados, por ejemplo, hemos visto que se usan videos de YouTube para mantener la atención y el interés del alumnado.

. Escena optimista: se encuentra centrada en la concepción curricular, se refiere a la relación existente entre la tecnología y los contenidos que van a ser enseñados, donde la intencionalidad pedagógica cobra especial importancia. En este escenario, la tecnología deberá estar incorporada en los proyectos curriculares planteándose preguntas como: ¿qué vamos a enseñar a través del uso de las tecnologías? ¿cómo se van a producir estos aprendizajes? ¿cuándo van a tener lugar los aprendizajes? Nuevamente, encontramos que esta 
escena también se ha visto reflejada durante la cuarentena, ya que la tecnología ha permitido tener una visión optimista de los contenidos que no pueden abordarse de forma presencial ya que muchos de los contenidos han sido adaptados y su forma de ser enseñados ha cambiado a formas mucho más visuales como videos, fotografías, videollamadas. Sin embargo, hemos observado que algunos contenidos no pueden trabajarse y ha sido necesaria una adaptación a la realidad virtual. Es importante aclarar que las tecnologías por sí solas nunca suplirán todo el proceso educativo.

. Escena de producción: esta escena se relaciona con la concepción creadora ya que, a través de la tecnología, el alumnado genera productos nuevos. En relación a los casos analizados, podemos afirmar que el nivel inicial se caracteriza por ser una etapa educativa especialmente imaginativa y fantasiosa. Por otro lado, cuando los alumnos producen y son protagonistas de sus propios aprendizajes, se fortalece el espíritu crítico, fomentando la participación en su cultura. En el caso de la institución de la Ciudad Autónoma de Buenos Aires, las maestras han creado foros dentro de la plataforma de "Aulas de Comunión" en los que los alumnos comparten sus trabajos, ideas y producciones. También observamos que en la institución de la ciudad de Ezeiza se ha fomentado la educación emocional y la creatividad desde elementos muy sencillos que se pueden encontrar en cualquier hogar.

- Escena problemática: se refiere a una concepción trialógica. Al hablar de tecnología, se tienen en cuenta tres factores que se relacionan entre sí y de los que dependen los unos de los otros: tecnología, alumno y adulto. Para Litwin (2008) en esta concepción, surge una escena problemática, en el mundo actual existe una gran influencia de los lenguajes audiovisuales que lleva a controversias y dilemas como por ejemplo que exista una presencia fuerte del alumno y del adulto en relación con las tecnologías. El adulto en ocasiones no domina el mundo digital y el niño posee una conexión especial con la tecnología en la que normalmente se ve beneficiada su autoestima.

Esta concepción, en relación a los casos analizados, se ha visto fortalecida en algunos ámbitos, pero en otros no se ha planteado una concepción trialógica. Por un lado, el uso de la tecnología 
durante la cuarentena, ha supuesto inicialmente una escena problemática por parte de todos los agentes implicados en las diferentes instituciones analizadas (debido a la falta de formación, al cambio inmediato, al uso de plataformas que se encontraban prácticamente en desuso o la incorporación de plataformas nuevas) y es posible que inicialmente se haya generado conflicto o tensión entre el equipo directivo, los maestros y las familias. Este escenario se ha visto modificado parcialmente a lo largo del tiempo y gracias al esfuerzo y al trabajo en equipo que se desarrolló al interior de cada institución analizada.

Tras analizar las cuatro escenas de relación que nos propone Litwin (2008), es interesante añadir una concepción democratizadora en relación al uso de las TIC en la enseñanza. Esta dimensión se centra en permitir un acceso realista de las tecnologías en el ámbito educativo. Supone hablar de equidad e inclusión de las tecnologías por parte de todo el alumnado, asegurando un aprendizaje igualitario para todos. Al vivir en la sociedad de la información y comunicación, es inviable pensar en educar a los alumnos desde una perspectiva de alfabetización digital. Este es un tema urgente, pero debido a la situación de pandemia global, adquiere aún más importancia, puesto que las tecnologías están presentes en todos los ámbitos de nuestra realidad cultural, social y económica. En relación con esta concepción, encontramos que la situación de pandemia global ha demostrado que aún falta mucho camino por recorrer, puesto que se ha visto que el acceso a las tecnologías aún no tiene un carácter inclusivo, tal como mencionamos anteriormente. No obstante, la UNESCO comenzó a aplicar otros proyectos de empoderamiento destinados a la población desfavorecida, en los que se observó que existían algunas dificultades atendiendo a la brecha digital. Por tanto, se trató de fomentar el uso de Internet, entre otras cosas, para compartir experiencias y recursos, así como medio de comunicación y aprendizaje social, favoreciendo el desarrollo de estas competencias en los sectores que presentan mayor dificultad en función de las situaciones en las que se encuentran. Por otro lado, la Agencia Española de Cooperación Internacional para el Desarrollo (AECID), utilizó su programa "Incorporación de las nuevas tecnologías para facilitar el acercamiento de la sociedad española al sur" para acercar dos campos de acción a través del uso de Internet: en primer lugar, las modalidades de aprendizaje electrónico y, en segundo lugar, la construcción de recursos virtuales. En este aspecto, se haría referencia a la 
dimensión de la planificación y administración escolar (Rosales Estrada, 2007, citado en Sevilla, et al., 2017, p. 142) ya que se trata de seleccionar con adecuación los recursos de aprendizaje (bien sean presenciales y/o virtuales) que se van a desarrollar.

\section{Conclusiones}

En base a lo expuesto en el presente artículo, podemos afirmar que en los tres casos analizados resultó fundamental la capacitación docente para poder lograr una inclusión profunda de las tecnologías en el Nivel Inicial en el contexto de cuarentena. Buckingham (2008) sostiene que la escuela es la responsable de abordar la tecnología, y en estos casos se observó que fue cada institución la que brindó la oportunidad de que los docentes se capaciten en torno a la temática para poder enseñar en esta situación repentina y no planificada.

Tenti Fanfani (2007) dice que el avance de las TIC presiona la formación y esta se vuelve ineludible. Por ello, se recomienda continuar con la formación intensiva sobre las TIC y las TAC para brindar saberes teóricos y prácticos que fomenten el uso adecuado de las tecnologías en el aula en contexto de cuarentena, y también para cuando se regrese a las clases presenciales.

La situación vivida ha sido algo excepcional e inédito para el mundo sin apenas precedentes, por lo que a pesar de los posibles errores y conflictos que se han generado durante este periodo de tiempo, se han tratado de solventar en la práctica de la mejor manera posible. Además, gracias a todas las aportaciones realizadas se han descubierto aspectos mejorables y posibles líneas de investigación y reflexión, que a futuro nos permitirán seguir avanzando hacia nuevos caminos que integren las nuevas tecnologías en nuestra vida diaria en las aulas, revisando los fallos cometidos para evitar repetirlos. Sin lugar a dudas, un nuevo camino educativo se abre paso ante nosotros, dejando paso a su vez a grandes interrogantes y cuestiones por abordar: ¿Qué enseñanzas nos deja lo vivido durante la cuarentena, acerca de cómo mejorar el Nivel Inicial? ¿Qué hemos aprendido sobre cómo incluir las tecnologías en dicho nivel? ¿Podremos producir mejoras a raíz de esta experiencia? 


\section{Referencias}

Aguerrondo, I. (2010). Enseñar y aprender en el siglo XXI. Seminario Desafíos para la Educación. Una mirada a diez años. Universidad Católica de Uruguay Montevideo. Disponible en http://www.uca.edu.ar/uca/common/grupo95/files/articulos-aguerrondo-ucamontev-2010-2-en-senar-y-aprender-en-el-siglo-xxi.pdf Consulta 4/6/2017

Aguilar Perera, M. V., \& Cuesta Suárez, H. (2009). Importancia de trabajar las TIC en educación infantil a través de métodos como la webquest. Revista Medio y Educación (34), 81-94.

Almirón, M.E. y Porro, S. (2014) Las TIC en la enseñanza: un análisis de casos. [Versión electrónica], Revista Electrónica de Investigación Educativa, 16 (2), 152-160.

Asorey, E., \& Gil Jesús. (2009). El placer de usar las TIC en el aula de infantil. Participación Educativa (12), 110-119.

Barberá, E. en el Prólogo de Kozak, D. (2010). "Escuela y Tics: los caminos de la innovación" Colección En las Aulas. (1 ${ }^{\mathrm{a}}$ ed.). Buenos Aires, Argentina. Lugar Editorial.

Batista, M.A, Celso, V.E y otros. (2007). Tecnología de la información y la comunicación en la escuela: trazos, claves y oportunidades para su integración pedagógica. Buenos Aires, Argentina. Ministerio de Educación, Ciencia y Tecnología.

Buckingham, D. (2008) "Repensar el aprendizaje en la era de la cultura digital". Dossier La Escuela y los Medios. "El Monitor de la Educación”. (n¹8) mes de septiembre de 2008. Ministerio de Educación, Ciencia y Tecnología de la Nación. 28-29. 
Cabero Almenara, J. (1996) “Nuevas tecnologías, comunicación y educación”. Edutec Revista Electrónica de Tecnología Educativa. (N¹) ([Versión digital] mes de febrero de 1996. Mallorca, España. 2.

Cabero, J. (2006). Bases pedagógicas del e-learning. Revista de la Universidad y Sociedad del Conocimiento (RUSC), Vol. 3, Núm. 1 (pp. 1-10)

Caro, M. I. (2017). La tecnología educativa y la pedagogía, dos elementos básicos en los procesos de enseñanza aprendizaje innovadores. En H. Sevilla, F. Tarasow, \& M. Luna, Educar en la era digital. Docencia, tecnología y aprendizaje (págs. 137-156). Guadalajara, México: Pandora.

Casablancas, S. (2014). Enseñar con tecnologías. Transitar las TIC hasta alcanzar las TAC. Colección didáctica "Caminos de tiza". Buenos Aires: Mandioca. Disponible en: http://www.silvinacasablancas.com/libro/Ensenar_con_tecnologias-Silvina_Casablancas.pdf

Chávez, P. R. (2017). Tecnologías para el aprendizaje y desafíos curriculares. En Sevilla, H., Tarasow, F., \& Luna, M. (Ed) Educar en la era digital. Docencia, tecnología y aprendizaje (pp. 95-119). Guadalajara, México: Pandora.

Coll, C., Mauri, T. y Onrubia, J. (2008). Análisis de los usos reales de las TIC en contextos educativos formales: una aproximación sociocultural. [Versión electrónica], Revista Electrónica de Investigación Educativa, 10 (1)

Coombs, P., Prosser, R. y Ahmed, M. (1973). New Paths To Learning for Rural Children and Youth. New York: International Council for Educational Development for UNICEF.

Cukierman, U. y otros. (2009). Tecnología educativa, recursos, modelo y metodologías. (1 ed.). Buenos Aires, Argentina. Pretince Hall. 
Díaz, F. (1993). El aprendizaje significativo desde una perspectiva constructiva. Revista Educar, núm. 4 (pp. 24-40)

Dussel, I. y Southwell, M. (2007). Lenguajes en plural. Revista El Monitor de la educación, $13,25-32$.

Echevarría, J. (2000). Educación y tecnologías telemática. s Revista Iberoamericana, 24, pp. $17-36$

Efron, G. (2010) “Cuando los medios aportan un valor agregado”, Dossier La Escuela y los Medios. "El Monitor de la Educación”. (n²4) mes de marzo de 2010. Ministerio de Educación, Ciencia y Tecnología de la Nación.32-33.

Ewen, S. (1998). Todas las imágenes del consumismo. La política del estilo en la cultura contemporánea. México: Grijalbo.

Flick, U. (2004). Introducción a la investigación cualitativa. (T. del Amo, Trad.). Madrid, España. Morata.

Foschi, R. (2020). María Montessori. Barcelona: Ediciones octaedro.

Gay, A. (1996). La cultura tecnológica y la Escuela. (2ª ed.). Córdoba, Argentina. Ediciones tec

Karsenti, T. y Lira, M. L. (2011). ¿Están listos los futuros profesores para integrar las TIC en el contexto escolar? El caso de los profesores de Quebec, Canadá. [Versión electrónica], Revista Electrónica de Investigación Educativa, 13(1), 56-70.

Litwin, E. y Otros (2005). Tecnologías educativas en tiempo de internet. ( $1^{\mathrm{a}}$ ed.) Buenos, Aires, Argentina. Amorrortu. 
Lugo, M. T. y Kelly V. (2011). El modelo 1 a 1: un compromiso con la calidad y la igualdad educativas. La gestión de las TIC en la escuela secundaria: nuevos formatos institucionales. [Versión digital]. Ministerio de Educación de la Nación. Buenos Aires, Argentina. Pág. 2021.

Luna Rizo, M., \& Ramos Zepeda, D. (2017). Acercamiento a las TIC y TAC en la capacitación docente. En H. Sevilla, F. Tarasow, \& M. Luna, Educar en la era digital. Docencia, tecnología y aprendizaje (págs. 181-201). Guadalajara, México: Pandora.

Miklos, T. (2012). "Prospectiva de la Educación virtual; el caso de América Latina". En Morocho, M. y Rama, C. (eds.) Las nuevas fronteras de la educación a distancia (pp. 115136). Loja, Ecuador: Universidad Técnica Particular de Loja.

Minzi, V. (2010). "Qué hay que saber de tecnología”. Dossier La Escuela y los Medios. "El Monitor de la Educación”. (n²5) mes de junio de 2010. Ministerio de Educación, Ciencia y Tecnología de la Nación. 43.

Moreno, M. (2012). "Educación a distancia, un caleidoscopio para el aprendizaje en la diversidad”. En: Moreno, M., Veinte visiones de la educación a distancia (pp. 17-30). Guadalajara: Universidad de Guadalajara.

Pastor-Homs, M. (2001). Orígenes y evolución del concepto de educación no formal. Revista española de pedagogía, Vol. 59, Núm. 220 (pp. 525-545)

Pettinari, A. (2020). Posteo en red social Facebook: Esto no es educación a distancia. Recuperado de https://www.facebook.com/ana.pettinari/posts/10158255546255948 Prensky, M. (2000). Digital Game - Based Learning. (1 ${ }^{\mathrm{a}}$ ed.). New York, USA. Mc Graw Hill.

Rolandi, A. (2015). Las tecnologías en las prácticas de enseñanza de los docentes de Nivel Inicial: análisis e interpretaciones sobre sus usos a partir de concepciones actuales del campo 
de la tecnología educativa. (Tesis doctoral publicada). Universidad de Buenos Aires: Facultad de Filosofía y Letras.

Salomon, G., Perkins, D. y Globerson, T. (1992). "Coparticipando en el conocimiento: la ampliación de la inteligencia humana con las tecnologías inteligentes". Revista Comunicación, lenguaje y educación. 13. Madrid.

Sancho, J. M. (2011). “Esperando a los otros”. Cuadernos de Pedagogía, 412, pp. 80-83.

Serrano Santoyo, A., \& Martínez, E. (2003). La brecha digital: mitos y realidades. Baja California, México: UABC.

Sevilla, H., Tarasow, F. y Luna, M. (coords.) (2017). Educar en la era digital. Docencia, tecnología y aprendizaje. Guadalajara: Pandora.

Tarasow, F. (2017). Educación en línea, el diseño en línea como hackeo. En Sevilla, H., Tarasow, F., \& Luna, M. (Ed) Educar en la era digital. Docencia, tecnología y aprendizaje. (pp. 95-119) Guadalajara, México: Pandora.

Tenti Fanfani, E. (2007) La condición docente. (1 $1^{\mathrm{a}}$ ed.) Buenos Aires, Argentina. Siglo XXI editores.

UNESCO (2005). Information and Communication Technologies (ICTS) for Community Empowerment through Non-Formal Education. Bankok, Thailand and Uzbekistan. Bangkik: UNESCO Asia and Pacific Regional Bureau for Education. 
REVISTA INTERNACIONAL DE PEDAGOGÍA E INNOVACIÓN EDUCATIVA |

Volumen 1. Número 2. Julio - Diciembre 2021 ISSN: 2745-0341 (En línea) 